\title{
Breakdown of staggered fermions at nonzero chemical potential
}

\author{
Maarten Golterman \\ Theory Group, Saha Institute of Nuclear Physics, 1/AF, Salt Lake, Kolkata 700064, Indid \\ Yigal Shamir and Benjamin Svetitsky \\ School of Physics and Astronomy, Raymond and Beverly Sackler \\ Faculty of Exact Sciences, Tel Aviv University, 69978 Tel Aviv, Israel
}

\begin{abstract}
The staggered fermion determinant is complex when the quark chemical potential $\mu$ is nonzero. Its fourth root, used in simulations with dynamical fermions, will have phase ambiguities that become acute when $\operatorname{Re} \mu$ is sufficiently large. We show how to resolve these ambiguities, but our prescription only works very close to the continuum limit. We argue that this regime is far from current capabilities. Other procedures require being even closer to the continuum limit, or fail altogether, because of unphysical discontinuities in the measure. At zero temperature the breakdown is expected when $\operatorname{Re} \mu \gtrsim m_{\pi} / 2$, where $m_{\pi}$ is the pion mass. Estimates of the location of the breakdown at nonzero temperature are less certain.
\end{abstract}

The direct study of quantum chromodynamics (QCD) at nonzero chemical potential $\mu$ faces the considerable hurdle presented by the complex measure in the euclidean path integral (for a review, see Ref. [1]). This requires the adoption of various stratagems in order to make Monte Carlo analysis possible. These stratagems include Taylor expansions around $\mu=0$ [2]; analytic continuation from the imaginary $\mu$ axis (where the measure is real and positive) [3]; and multiparameter reweighting using real measures that push the complex phases into the observables [4, 5, 6, 7, 8]. All these methods envisage a well-defined path integral with complex measure at real, nonzero values of $\mu$. While the first two methods never actually calculate fermion determinants for $\operatorname{Re} \mu \neq 0$, the third method does calculate ratios of these determinants as configurations are analyzed. For a different reweighting method based on the canonical ensemble, see Ref. [9].

Calculations at $\mu \neq 0$ are often performed with staggered fermions. These are contained in a singlecomponent fermion field that makes for efficient calculation of the fermion determinant and Green functions; a further advantage is a chiral symmetry that is only broken softly by the mass parameter $m$. A disadvantage of staggered fermions is residual doubling, wherein a single lattice field actually represents four physical flavors (nowadays called tastes) that become degenerate in the continuum limit. All these tastes affect the dynamics of the theory, as is easily seen by considering fermion loops in a diagrammatic expansion of the determinant. In order to represent a single flavor of fermion with mass $m$, then, one takes the fourth root of the determinant. Thus one hopes to arrive at two light flavors and one strange quark in the continuum limit.

\footnotetext{
*Permanent address: Department of Physics and Astronomy, San Francisco State University, San Francisco, CA 94132, USA
}

The QCD partition function on a lattice, with two light quarks plus the strange quark, is given by

$$
\begin{aligned}
Z=\int \mathcal{D} U & e^{-S_{g}(U)} \operatorname{det}\left[D(U)+m_{l}\right]^{1 / 2} \\
& \times \operatorname{det}\left[D(U)+m_{s}\right]^{1 / 4}
\end{aligned}
$$

where $D(U)$ is the staggered-fermion hopping matrix, including factors of $\exp ( \pm \mu)$ on the timelike links; $m_{l}$ is the common mass of the two light quarks and $m_{s}$ is the strange quark mass. When $\operatorname{Re} \mu=0$, the matrix $D(U)$ is anti-hermitian. The eigenvalues of $D+m$ then come in conjugate pairs, $\eta=m \pm i E$, so the determinant is real and strictly positive (for $m \neq 0$ ). If we take the fourth root to be real and positive as well, the measure in $Z$ is a continuous functional of the gauge field $U$. We will assume here that taking the fourth root is a valid procedure for $\mu=0$ [10, 11, 12].

When $\operatorname{Re} \mu \neq 0$, on the other hand, the spectrum of $D+m$ spreads out into the complex plane and the determinant becomes complex. Taking the fourth root of the complex determinant introduces phase ambiguities. A prescription must be found for resolving these ambiguities. The problem first appears when $\mu \gtrsim m$, when rare configurations admit eigenvalues near zero. At some larger value of $\mu$, near-zero eigenvalues become common in typical configurations. Analysis of the $\epsilon$-regime suggests that in the zero-temperature theory this happens rather abruptly when $\mu \approx m_{\pi} / 2[13]$; at $T>0$ this point is believed to move towards higher $\mu$ [14].

We present below a sensible prescription for taking the fourth root when $\operatorname{Re} \mu \neq 0$. This prescription requires that we work near the continuum limit. The breakdown of the method on coarser lattices, in fact for any realistic value of the lattice spacing, is quite general and indicative as well of the failure of alternative rules for dealing with the fourth root. The discussion carries over to the square root used for the two light quarks. 
An unambiguous definition of the phase of the fourth root requires understanding taste symmetry and its breaking at finite lattice spacing. To this end, let us describe the IR and UV regimes of the complex spectrum of $D+m$ near the continuum limit (see Fig. 1). The

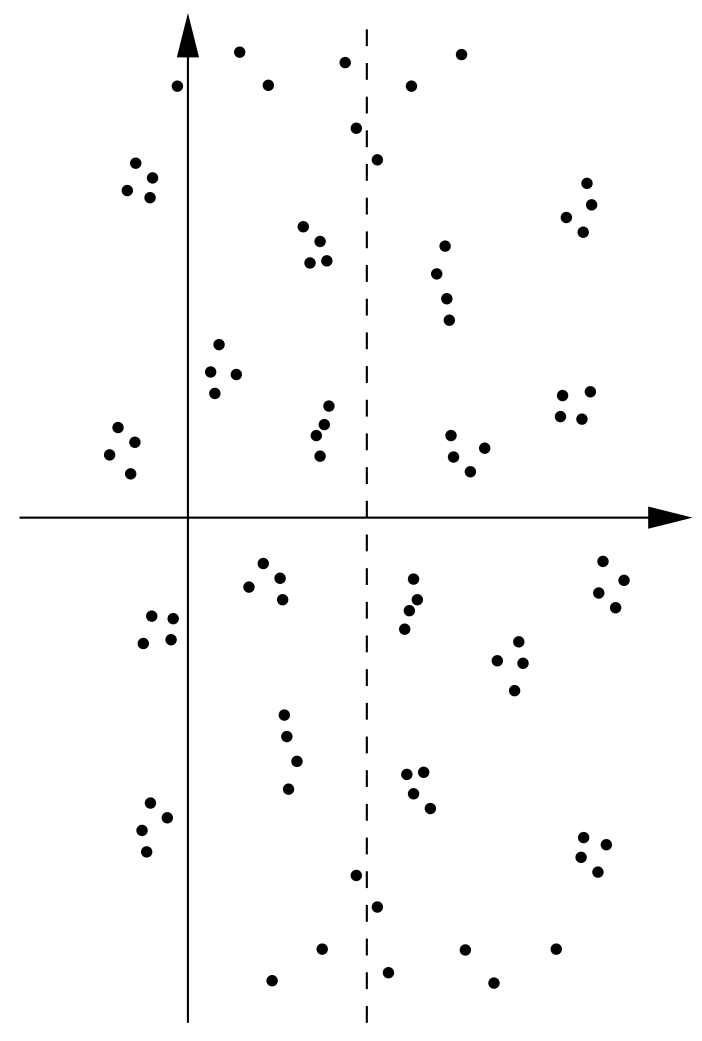

FIG. 1: Sketch of complex eigenvalues $\eta_{i}$ of $D+m$ when $\operatorname{Re} \mu$ is somewhat larger than $m_{\pi} / 2$. The dashed line is $\operatorname{Re} \eta=m$. Eigenvalues closest to the real axis are clustered into quartets with splittings of $O\left(a \Lambda^{2}\right)$; typical spacing between quartets is $\propto V^{-1 / 2}$ (see text). Farther from the real axis the quartet structure disappears. The eigenvalues come in pairs $(\eta, 2 m-$ п) [15].

eigenvalues $\eta_{i}$ that fall in the IR regime will form approximately degenerate, well distinguished quartets, as dictated by taste symmetry. These will include eigenvalues near the QCD scale, $\left|\eta_{i}\right| \approx \Lambda$. For UV eigenvalues, with $\left|\eta_{i}\right| \rightarrow a^{-1}$, large taste violations are unavoidable. Since, however, both $m$ and $\mu$ are now small compared to $\left|\eta_{i}\right|$, these eigenvalues are dominated by the anti-hermitian $D(\mu=0)$, and so their phases should be near $\pm \pi / 2$, with deviations that are $O(a m, a \mu)$. We expect that there will be a large overlap region where the eigenvalues $\eta_{i}$ satisfy both conditions: they should fall into approximately degenerate quartets and their phases will be near $\pm \pi / 2$.

Now the prescription for the fourth root of the determinant is straightforward. For the IR quartets, we proceed one quartet at a time, and we take the fourth root of their product to have a phase angle equal to the phase of the center of the quartet. Thus we construct a single-flavor theory, with one eigenvalue in place of each quartet. For the UV eigenvalues, ${ }^{1}$ there is no need for subtlety; since the eigenvalues lie near the imaginary axis, we can take the arguments of their fourth roots to lie near $\pm \pi / 8$.

The fourth root so constructed will usually be a welldefined, continuous functional of the gauge field, with a slowly varying phase angle. An exception will arise if an eigenvalue quartet comes too close to the origin. If, in fact, the four eigenvalues frame the origin, the average phase of the quartet becomes meaningless and any definition will fluctuate rapidly as the eigenvalues move. Such an occurrence will, however, be rare. The typical splitting $\delta$ within a quartet will be ${ }^{2} O\left(a \Lambda^{2}\right)$, and so the probability of finding an eigenvalue this close to zero is proportional to $a^{2}$. A more detailed estimate follows.

First, let us discuss orders of limits. At $\mu=0$, the relative taste splittings inside a quartet, $\left|\eta_{i}-\eta_{j}\right| /|\bar{\eta}|$, are bounded from above by $a \Lambda^{2} / \mathrm{m}$. If we want the taste splittings of the pseudo-Goldstone bosons to be small, we must take the continuum limit before the chiral $(m \rightarrow 0)$ limit [16]. Let us now assume $\operatorname{Re} \mu \sim \Lambda$. The eigenvalue density near the origin in the complex $\eta$ plane scales like $V \Lambda^{2}$ with the volume $V$ of the lattice. Thus $m$ gets replaced by the $O\left[(\sqrt{V} \Lambda)^{-1}\right]$ mean distance between quartets (the same estimate applies to the typical magnitude of the eigenvalue nearest to the origin), and $\left|\eta_{i}-\eta_{j}\right| /|\bar{\eta}|$ comes out to be $O\left(a \sqrt{V} \Lambda^{3}\right)$ for the near-zero quartets. Now it is the product $a \sqrt{V} \Lambda^{3}$ that must remain small for the sake of good taste. If not, the splittings within a quartet will be as large as the splittings between different quartets, and our prescription will break down. When approaching the continuum limit, the volume must be limited such that $a \sqrt{V} \Lambda^{3} \rightarrow 0$ as well.

With the eigenvalue density estimated as above, the fraction of quenched configurations containing an eigenvalue quartet that frames the origin is $O\left(a^{2} V \Lambda^{6}\right)$. This, then, is the order of the error introduced by either taking or dropping these configurations. Reweighting by the determinant provides further suppression by $O\left(a \sqrt{V} \Lambda^{3}\right)$; this is the ratio of fourth roots of the said eigenvalue quartet and of a typical near-zero quartet. The systematic error thus becomes $O\left[\left(a \sqrt{V} \Lambda^{3}\right)^{3}\right]$.

Is it really necessary to extract individual eigenvalues and to group them into quartets? The procedure appears cumbersome, but other prescriptions that come to mind

\footnotetext{
${ }^{1}$ Renormalization-group analysis [1] suggests that the log of the UV part of the determinant contributes only a local correction to the effective action of the gauge field; it can even be discarded, if one compensates by renormalizing the gauge coupling.

2 This can be seen from the representation of the staggered operator in taste basis, where the taste breaking comes from a dimension-5 operator that shifts eigenvalues within a quartet as $\eta \rightarrow \eta \pm \delta$, where $\delta$ is fixed and $O(a)$. This is consistent with the usual picture of $O\left(a^{2}\right)$ discretization errors because the product of eigenvalues will shift by $O\left(\delta^{2}\right)$.
} 
produce larger errors close to the continuum limit, or even fail altogether. If one were to calculate the determinant $\mathcal{D}$ in full and only then to take its fourth root $\Delta$ in the cut plane $|\operatorname{Arg} \mathcal{D}|<\pi$, the result would be restricted to the wedge $|\operatorname{Arg} \Delta|<\pi / 4$. This is inconsistent with an ordinary single-flavor theory, whose determinant is free to wander the entire complex plane.

If one were to take the fourth root of each individual $\eta_{i}$ in the cut plane before multiplying them together, there would be frequent jumps of $\pi / 2$ in the phase of $\Delta$ as single eigenvalues cross the cut. Here the systematic error can be estimated: It would be $O\left(a V \Lambda^{5}\right)$, which is the fraction of configurations in which a quartet is bisected by the negative real axis, close to the continuum limit. [The probability that none of the $O\left(V \Lambda^{4}\right)$ IR eigenvalues fall in a strip of width $a \Lambda^{2}$ along the negative real axis scales like $(1-a \Lambda)^{V \Lambda^{4}} \approx 1-a V \Lambda^{5}$.] This estimate is only valid if one has verified the existence of well-defined quartets in all configurations. Moreover, this error is can be fatally large even if the figure of merit $a \sqrt{V} \Lambda^{3}$ is small.

Are current calculations anywhere near to realizing the quartet structure of IR eigenvalues that will make calculation of the fourth root consistent? We suspect that the answer is negative. Simulations at $\mu=0$ show nice taste degeneracy in the lowest eigenvalues only when the lattice cutoff is $a^{-1} \approx 2 \mathrm{GeV}$ or larger, and only if highly improved gauge and staggered actions are used [12]. The state of affairs is much less satisfactory in existing simulations with $\operatorname{Re} \mu>0$, where the cutoff is at $1 \mathrm{GeV}$ or less. Even in the $\mu=0$ simulations, significant taste violations will reappear if one looks beyond the lowest few quartets. While this does not hamper simulations at $\mu=0$, for the $\mu>0$ case we do require a distinct quartet structure all the way up to $|\eta| \sim \Lambda$ and beyond.

In general terms, what would it take to define a fourth root that is single-valued and continuous? Consider a closed trajectory in gauge-field space that avoids the zeros of the fermion determinant $\mathcal{D}$. When this trajectory is mapped into the complex $\mathcal{D}$ plane, the winding number around the origin must be a multiple of 4 . In practice, this will never be guaranteed, precisely because of tastesymmetry breaking. We believe that current simulations are plagued by eigenvalues with no particular multiplet structure. Problems will arise when any single eigenvalue circles the origin. Depending on the treatment given the fourth root, either the eigenvalue will cross a cut, causing a jump in the fourth root; or the eigenvalue will move onto another Riemann sheet, giving a multi-valued root.

[We note that, for $m=0$, two eigenvalues will always circle the origin simultaneously, because they appear in $(\eta,-\eta)$ pairs in that case (see Fig. 11). It would then be possible to define a square root by requiring continuity, because the winding number of the determinant around the origin would always be even. This only works, however, for $m=0$, and it will not help for the fourth root.]

We illustrate this problem in two dimensions. We shall exhibit eigenvalues near the origin for selected gauge fields and show how small changes in the gauge field can take a single eigenvalue around the origin and thus through any cut that can be defined. The staggered fermion action on a lattice of $L_{t} \times L_{x}$ sites is

$$
\begin{aligned}
S= & \bar{\psi}(D+m) \psi \\
= & \frac{1}{2} \sum_{t=1}^{L_{t}} \sum_{x=1}^{L_{x}}\left[\bar{\psi}_{t, x} U_{t, x}^{0} \psi_{t+1, x}-\bar{\psi}_{t+1, x} U_{t, x}^{0 \dagger} \psi_{t, x}\right. \\
& +(-1)^{t}\left(\bar{\psi}_{t, x} U_{t, x}^{1} \psi_{t, x+1}-\bar{\psi}_{t, x+1} U_{t, x}^{1 \dagger} \psi_{t, x}\right) \\
& \left.+2 m \bar{\psi}_{t, x} \psi_{t, x}\right] .
\end{aligned}
$$

A change of variables 17] has removed the chemical potential from all links of the lattice except for those that connect $t=L_{t}$ to $t=1$. First we choose a gauge field that is zero except for a constant Polyakov loop $U$ on the last time-link. This is equivalent to writing the free operator with the modified antiperiodic boundary conditions

$$
\begin{aligned}
\psi_{t, L_{x}+1} & \equiv-\psi_{t, 1}, & \psi_{L_{t}+1, x} & \equiv-U e^{L_{t} \mu} \psi_{1, x} \\
\bar{\psi}_{t, L_{x}+1} & \equiv-\bar{\psi}_{t, 1}, & \bar{\psi}_{L_{t}+1, x} & \equiv-\bar{\psi}_{1, x} U^{\dagger} e^{-L_{t} \mu}
\end{aligned}
$$

\section{A Fourier transformation from $x$ to $p$ gives}

$$
\begin{gathered}
S=\sum_{t=1}^{L_{t}} \sum_{p}\left\{\frac{1}{2}\left(\bar{\psi}_{t, p} \psi_{t+1, p}-\bar{\psi}_{t+1, p} \psi_{t, p}\right)\right. \\
\left.\quad+\left[m+(-1)^{t} i \hat{p}\right] \bar{\psi}_{t, p} \psi_{t, p}\right\} \\
\equiv \sum_{t, t^{\prime}} \sum_{p} \bar{\psi}_{t, p} M_{t, t^{\prime}}(p) \psi_{t^{\prime}, p},
\end{gathered}
$$

where $\hat{p}=\sin p$. The momentum takes the values $p_{n}=(2 n+1) \pi / L_{x}$. We must keep careful track of multiplicities. If we take $L_{x}$ to be a multiple of four, then none of the momenta $p=0, \pi / 2, \pi,-\pi / 2$ are in the spectrum. This assures that each possible value of $\sin ^{2} p$ has a multiplicity of four. The fermion matrix in the momentum basis is given explicitly by 


$$
M(p)=\left(\begin{array}{ccccccc}
m-i \hat{p} & \frac{1}{2} & 0 & 0 & \cdots & 0 & \frac{1}{2} e^{i \phi-L_{t} \mu} \\
-\frac{1}{2} & m+i \hat{p} & \frac{1}{2} & 0 & \cdots & 0 & 0 \\
0 & -\frac{1}{2} & m-i \hat{p} & \frac{1}{2} & \cdots & 0 & 0 \\
0 & 0 & -\frac{1}{2} & m+i \hat{p} & \cdots & 0 & 0 \\
\vdots & \vdots & \vdots & \vdots & \ddots & \vdots & \vdots \\
0 & 0 & 0 & 0 & \cdots & m-i \hat{p} & \frac{1}{2} \\
-\frac{1}{2} e^{-i \phi+L_{t} \mu} & 0 & 0 & 0 & \cdots & -\frac{1}{2} & m+i \hat{p}
\end{array}\right)
$$

We have chosen $U$ diagonal, whereupon the determinant factors into $N_{c}$ color factors, and selected one of the colors.

$M$ is not anti-hermitian; it satisfies instead $M^{\dagger}(m, \mu)=-M\left(-m,-\mu^{*}\right)$. The right-eigenvectors of $M$ may be found via an alternating ansatz,

$$
\psi_{t}= \begin{cases}\alpha e^{i \varnothing t}, & t \text { odd } \\ \beta e^{i \varnothing t}, & t \text { even }\end{cases}
$$

We find the eigenvalues

$$
\eta_{ \pm}=m \pm i \mathcal{E}
$$

where $\mathcal{E}=\sqrt{\hat{p}^{2}+\hat{\omega}^{2}}$, with $\hat{\omega} \equiv \sin \omega$. The boundary conditions lead to the quantization of $\operatorname{Re} \varnothing$, viz.,

$$
\operatorname{Re} \emptyset_{n}=\operatorname{Im} \mu+\frac{(2 n+1) \pi-\phi}{L_{t}},
$$

and further require

$$
\operatorname{Im} \varnothing_{n}=-\operatorname{Re} \mu .
$$

The eigenvalues depend on $p$ only via $\hat{p}^{2}=\sin ^{2} p$, and thus the complete set of eigenvalues shows a four-fold degeneracy.

When $\operatorname{Re} \mu \gtrsim m$ [more precisely, $\cosh (2 \operatorname{Re} \mu)>1+$ $2 \mathrm{~m}^{2}$ ], the eigenvalue $\eta_{+}$can be zero if $\mathcal{E}$ is pure imaginary, i.e., $\mathcal{E}=i m$. This can be accomplished by giving the Polyakov loop $U$ a value corresponding to

$$
\phi=L_{t} \operatorname{Im} \mu+\pi \quad(\bmod 2 \pi)
$$

the zero eigenvalue is then found by choosing $p$ appropriately and taking $n=0$ so that $\operatorname{Re} \omega=0$. A small change in $U$ from this value gives us a four-fold degenerate eigenvalue near but not at zero; in fact, $p$ cannot be chosen to give an exact zero in the first place unless $L_{x}$ is infinite.

We can add a gauge field to break the degeneracy by pushing the four eigenvalues in different directions. Consider a spatial gauge field that, for all $x$, takes values $U_{o}^{1}=e^{i A_{o}}$ and $U_{e}^{1}=e^{i A_{e}}$ for odd and even $t$ respectively. (We assume that these spatial links commute with the constant Polyakov loop.) In Eq. (44), this replaces $\hat{p}=\sin p$ by $\hat{p}_{o} \equiv \sin \left(p+A_{o}\right)$ and by $\hat{p}_{e} \equiv \sin \left(p+A_{e}\right)$ for

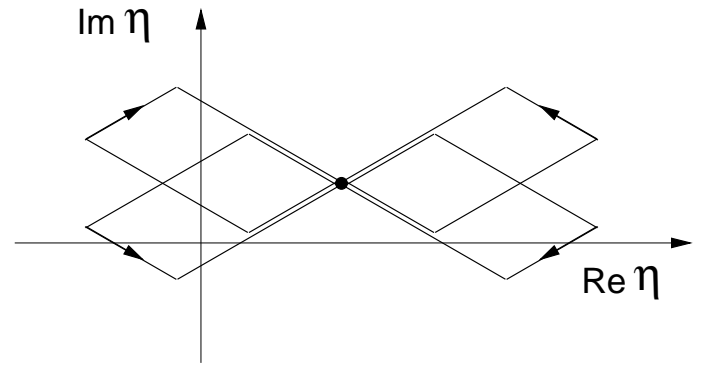

FIG. 2: Breaking of the fourfold degeneracy and transport of one eigenvalue around the origin. The small filled circle marks the original (fourfold) eigenvalue $\eta$. The two upper trajectories have been slightly displaced for clarity.

odd and even $t$ respectively. The spectrum can again be calculated exactly. The new eigenvalues are

$$
\eta_{ \pm}^{\prime}=m+i\left(\hat{p}_{e}-\hat{p}_{o}\right) / 2 \pm i \mathcal{E}^{\prime},
$$

where $\mathcal{E}^{\prime}=\sqrt{\left(\hat{p}_{o}+\hat{p}_{e}\right)^{2} / 4+\hat{\omega}^{2}}$. The conditions (7) and (8) on $\omega$ are unchanged. If we expand to first order in $A_{o}$ and $A_{e}$, we obtain $\eta_{ \pm}^{\prime}=\eta_{ \pm}+\delta \eta_{ \pm}$, where

$$
\delta \eta_{ \pm}=\frac{i \cos p}{2}\left[\left(A_{e}-A_{o}\right) \pm \frac{\sin p}{\mathcal{E}}\left(A_{o}+A_{e}\right)\right] .
$$

Equation (11) contains both $\cos p$ and $\sin p$. This means that the four-fold degeneracy among $p,-p, \pi+p$ and $\pi-p$ is completely lifted by the gauge field (see Fig. 2) ${ }^{3}$

To see how we can make a single eigenvalue circle the origin as in Fig. 2 let us begin again from the degenerate case. The eigenvalue quartet closest to zero will be of order $1 / L_{x} \ll 1$. We turn on $A_{o}$, then $A_{e}$, and then turn them off in the same order. The closed trajectory of only one eigenvalue circles the origin once; hence also the determinant circles the origin once.

\footnotetext{
${ }^{3}$ When the gauge field is zero the fourfold degeneracy is a result of taste symmetry plus charge conjugation. The constant gauge field $A_{e}=A_{o}$ breaks the latter, leaving the degeneracy between $p$ and $p+\pi$. Only when $A_{e} \neq A_{o}$ is the taste symmetry broken, as can be shown with the method of Ref. [18].
} 
A local fluctuation in the gauge field can accomplish the same lifting of the degeneracy and transport of eigenvalues. For instance, beginning with the degenerate case, choose a single spacelike link at $(t, x)$ and set $U_{t, x}^{1}=1+i A$, with $A$ chosen to be diagonal in color. The first-order change in a zero eigenvalue is

$$
\delta \eta=\frac{i A \cos p}{L_{x} L_{t}} \times\left\{\begin{array}{rr}
-1-\frac{i}{m} \sin p, & t \text { odd } \\
1-\frac{i}{m} \sin p, & t \text { even }
\end{array}\right.
$$

Again, the result depends on both $\cos p$ and $\sin p$, so that the four-fold degeneracy is lifted.

Our study of two-dimensional QCD exhibits nondegenerate eigenvalues and shows how they wind around the origin. The fourth root of the determinant will be problematic here; the same will apply to any representation of the determinant as a product,

$$
\operatorname{det}(D+m)=\prod_{i} \xi_{i}
$$

where the factors are complex functions of the gauge field, if the fourth root of the determinant is taken by multiplying the fourth roots of the individual $\xi_{i}$ 's.

An example is the formula used by Fodor and Katz [5, 8], where $\xi_{i}=e^{L_{t} \mu}-\lambda_{i}$; the $\lambda_{i}$ are eigenvalues of a non-hermitian matrix on a three-dimensional sublattice. In our two-dimensional example, again one finds that by turning on the gauge field $A_{e, o}$ the degeneracy of the $\lambda_{i}$ is completely lifted.

The method of Ref. [5] guarantees continuity of the fourth root of each $\xi_{i}$ as a function of $\mu$, starting from a point where $\operatorname{Re} \mu=0$. This is not the same as continuity as a function of the gauge field, which, as we have seen, is the central issue. Each $\xi_{i}$ carries within it a tangle of information coming from all length scales; we do not even know if the $\xi_{i}$ 's will fall into well-distinguished quartets close to the continuum limit. If they do, it would follow as above that the systematic error of this prescription is due to $\xi_{i}$ 's located a distance of order $a$ from the negative real axis. As noted, this gives a systematic error of $O\left(a V \Lambda^{5}\right)$. If there are no quartets then the error will be larger.

We can make our estimates semi-quantitative for the simulation parameters of Ref. [8]. It is convenient to identify the QCD scale $\Lambda$ with the critical temperature $T_{c}$ at $\mu=0$. Since the temporal size of the lattices used was in the neighborhood of $1 / T_{c}$, in these units the lattice spacing was $a \approx 1 / L_{t}=1 / 4$; the largest volume used was $(12 / 4)^{3}=27$. As we have seen, if the eigenvalue spectrum is to exhibit a taste-quartet structure then the figure of merit $a \sqrt{V} \Lambda^{3}$ must be small. For these parameters, however, we arrive at a value of 1.3. This returns us to a picture of no quartet structure and hence an intractable error due to phase discontinuities.

The remaining question regarding Ref. [8] is whether the values of $\mu$ studied put the theory into the dangerous regime where the distribution of eigenvalues $\eta_{i}$ or $\xi_{i}$ reaches the origin. In fact $\operatorname{Re} \mu$ was varied up to and beyond the putative critical end point at $\mu_{c} \simeq 120 \mathrm{MeV}{ }^{4}$ This is well above the value of $m_{\pi} / 2$ for the realistic light-quark masses used, but, as we have noted, the critical value of $\mu$ is probably larger when $T>0$. The fact that the pseudo-critical temperature $T_{c}(\mu)$ determined in Ref. 8] agrees over a substantial interval with that calculated via analytic continuation $[\underline{3}$ is indicative of such a shift, perhaps as far as $\mu \simeq 100 \mathrm{MeV}$. Direct study of the spectral density would settle this question.

Let us summarize our findings. While there are new complications, taking the fourth root when $\operatorname{Re} \mu \neq 0$ could in principle be on a footing similar to that of the $\mu=0$ case. A requirement is that one work close enough to the continuum limit and set the volume such that taste multiplets have reappeared in the eigenvalue spectrum all the way up to $|\eta| \sim \Lambda$ and beyond. If one tracks the quartets and assigns their phases carefully, then configurations with quartets that frame the origin will bring a systematic error of $O\left[\left(a \sqrt{V} \Lambda^{3}\right)^{3}\right]$. If one calculates fourth roots of individual eigenvalues and ignores their quartet structure, then quartets that are bisected by the cut cause an error of $O\left(a V \Lambda^{5}\right)$. In practice, however, the demonstration of the necessary quartet structure appears to be far beyond any realistic simulation even at $\operatorname{Re} \mu=0$, let alone when $\operatorname{Re} \mu \neq 0$.

In simulations done at purely imaginary $\mu[\underline{3}$ the validity of the fourth root is on the same footing as at $\mu=0$, provided the continuum extrapolation is done before attempting the analytic continuation in $\mu$. Otherwise, on a finite lattice, the integration over the compact gauge field commutes with the analytic continuation in $\mu$. The method would then be tantamount to some prescription for taking the fourth root of the determinant on the individual configurations. The nature of this prescription, and its relation to the ones compared here, is beyond the scope of this paper.

\section{Acknowledgments}

We thank Philippe de Forcrand for stimulating our interest in this subject and for his comments. We also thank Zoltan Fodor and Kim Splittorff for timely and helpful correspondence and much discussion. We would like to thank the organizers of the Cyprus Workshop on Computational Hadron Physics (September 2005), where this work was initiated. MG thanks the physics departments at Tel Aviv University and the University of Rome "La Sapienza" as well as the Institute for Mathematical Sciences in Chennai for hospitality. This work was supported by the Israel Science Foundation under grant no. 173/05 and by the US Department of Energy.

\footnotetext{
4 This is the quark chemical potential; Ref. [8] gives the baryon chemical potential, $\mu_{B} \equiv 3 \mu$.
} 
[1] O. Philipsen, Proc. Sci. LAT2005, $016 \quad$ (2005) hep-lat/0510077.

[2] R. V. Gavai and S. Gupta, Phys. Rev. D 68, 034506 (2003) hep-lat/0303013; ibid. 71, 114014 (2005) hep-lat/0412035; C. R. Allton et al., ibid. 68, 014507 (2003) hep-lat/0305007; ibid. 71, 054508 (2005) hep-lat/0501030; S. Choe et al., Phys. Rev. D 65, 054501 (2002); I. Pushkina et al. [QCD-TARO Collaboration], Phys. Lett. B 609, 265 (2005) hep-lat/0410017.

[3] P. de Forcrand and O. Philipsen, Nucl. Phys. B 642, 290 (2002) hep-lat/0205016; ibid. 673, 170 (2003) hep-lat/0307020; M. D'Elia and M. P. Lombardo, Phys. Rev. D 67, 014505 (2003) hep-lat/0209146; ibid. 70, 074509 (2004) hep-lat/0406012; V. Azcoiti, G. Di Carlo, A. Galante and V. Laliena, J. High Energy Phys. 0412, 010 (2004) hep-lat/0409157; Nucl. Phys. B 723, 77 (2005) hep-lat/0503010.

[4] I. M. Barbour et al., Phys. Rev. D 56, 7063 (1997) hep-lat/9705038 and references therein.

[5] Z. Fodor and S. D. Katz, Phys. Lett. B 534, 87 (2002) hep-lat/0104001; J. High Energy Phys. 0203, 014 (2002) hep-lat/0106002.

[6] C. R. Allton et al., Phys. Rev. D 66, 074507 (2002) hep-lat/0204010.

[7] Z. Fodor, S. D. Katz and K. K. Szabo, Phys. Lett. B 568, 73 (2003) hep-lat/0208078; F. Csikor et al., J. High Energy Phys. 0405, 046 (2004) hep-lat/0401016.

[8] Z. Fodor and S. D. Katz, J. High Energy Phys. 0404, 050 (2004) hep-lat/0402006; Z. Fodor, S. D. Katz, K. K. Szabo and A. I. Toth, Nucl. Phys. Proc. Suppl. 140, 508 (2005) hep-lat/0410032.

[9] S. Kratochvila and P. de Forcrand, Nucl. Phys. Proc.
Suppl. 140, 514 (2005) hep-lat/0409072; Proc. Sci. LAT2005, 167 (2005) hep-lat/0509143; P. de Forcrand and S. Kratochvila, Nucl. Phys. Proc. Suppl. 153, 62 (2006) arXiv:hep-lat/0602024.

[10] C. Aubin and C. Bernard, Phys. Rev. D 68, 034014 (2003) hep-lat/0304014.

[11] Y. Shamir, Phys. Rev. D 71, 034509 (2005) hep-lat/0412014; Proc. Sci. LAT2005, 240 (2005) hep-lat/0509163 ; C. Bernard et al., Proc. Sci. LAT2005, 114 (2005) hep-lat/0509176.

[12] E. Follana, A. Hart and C. T. H. Davies [HPQCD Collaboration], Phys. Rev. Lett. 93, 241601 (2004) hep-lat/0406010; S. Dürr, C. Hoelbling and U. Wenger, Phys. Rev. D 70, 094502 (2004) hep-lat/0406027; K. Y. Wong and R. M. Woloshyn, ibid. 71, 094508 (2005) hep-lat/0412001; E. Follana, A. Hart, C. T. H. Davies and Q. Mason [HPQCD Collaboration], ibid. 72, 054501 (2005) hep-lat/0507011.

[13] I. Barbour et al., Nucl. Phys. B 275, 296 (1986); D. Toublan and J. J. M. Verbaarschot, Int. J. Mod. Phys. B 15, 1404 (2001) hep-th/0001110; G. Akemann, J. C. Osborn, K. Splittorff and J. J. M. Verbaarschot, Nucl. Phys. B 712, 287 (2005) hep-th/0411030.

[14] K. Splittorff, hep-lat/0505001 also talk given Lattice 2006, Tucson, Arizona, 23-28 July 2006.

[15] J. Smit and J. C. Vink, Nucl. Phys. B 286, 485 (1987).

[16] C. Bernard, Phys. Rev. D 71, 094020 (2005) hep-lat/0412030.

[17] A. Roberge and N. Weiss, Nucl. Phys. B 275, 734 (1986).

[18] M. Golterman and J. Smit, Nucl. Phys. B 245, 61 (1984). 